\title{
HYPONORMALITY OF TOEPLITZ OPERATORS ON THE BERGMAN SPACE OF AN ANNULUS
}

\author{
HOUCINE SADRAOUI AND MOHAMMED GUEDIRI
}

\begin{abstract}
A bounded operator $S$ on a Hilbert space is hyponormal if $S^{*} S-$ $S S^{*}$ is positive. In this work we find necessary conditions for the hyponormality of the Toeplitz operator $T_{f+\bar{g}}$ on the Bergman space of the annulus $\{1 / 2<|z|<1\}$, where $f$ and $g$ are analytic and $f$ satisfies a smoothness condition.
\end{abstract}

\section{INTRODUCTION}

A bounded operator $S$ on a Hilbert space is hyponormal if $S^{*} S-S S^{*}$ is positive. Hyponormality of Toeplitz operators has been studied by many authors. Hyponormality of these operators on the Hardy space was considered in [3, 4]. Hyponormality of these operators with a symbol of the form $g_{1}+\overline{g_{2}}$ on the Bergman space of the unit disk was first considered in [8]. Therein a necessary condition was proved, which was later improved in [1. Some special cases are treated in [7]. A sufficient condition when $g_{1}$ is a monomial and $g_{2}$ is a polynomial is proved in [9]. An improvement of the necessary condition in the case when $g_{1}$ and $g_{2}$ are binomials is given in [5]. Basic material on Toeplitz operators on the Bergman space of the unit disk can be found in [2]. In this work we consider hyponormality of Toeplitz operators on the Bergman space of an annulus.

We start with definitions and notations. Denote by $A_{1 / 2}^{2}$ the space of holomorphic functions on the annulus $C_{1 / 2}=\{z \in \mathbb{C}: 1 / 2<|z|<1\}$ such that $\int|h|^{2} d m(z)<\infty$, where $d m(z)=(4 / 3 \pi) d \lambda(z)$ and $\lambda$ is the Lebesgue measure on the annulus. If $h \in A_{1 / 2}^{2}$ we write $h=a_{0}+\sum_{1}^{\infty} a_{n} z^{n}+a_{-n} z^{-n}$ and we have $\|h\|^{2}=\sum_{0}^{\infty} \frac{4\left(1-(1 / 2)^{2 n+2}\right)}{3(n+1)}\left|a_{n}\right|^{2}+\frac{8}{3} \ln 2\left|a_{-1}\right|^{2}+\sum_{2}^{\infty} \frac{4\left(2^{2 n-2}-1\right)}{3(n-1)}\left|a_{-n}\right|^{2}$. We denote by $L^{2}\left(C_{1 / 2}\right)$ the space of measurable and square integrable functions with respect to $d m$ on $C_{1 / 2}$. Toeplitz operators on $A_{1 / 2}^{2}$ are defined by $T_{f}(h)=P(h f)$, where $f$ is bounded and measurable on $C_{1 / 2}, P$ is the orthogonal projection on

2010 Mathematics Subject Classification. Primary 47B35, 47B20; Secondary 15B48.

Key words and phrases. Toeplitz operator; Bergman space of an annulus; hyponormal; positive matrix.

The authors would like to extend their sincere appreciation to the Deanship of Scientific Research at King Saud University for its funding of this work through the Research Group project No. RG-1435-069. 
$A_{1 / 2}^{2}$, and $h$ is in $A_{1 / 2}^{2}$. The Hankel operators on the space $A_{1 / 2}^{2}$ are defined by $H_{f}(h)=(I-P)(h f)$. The space $A_{1 / 2}^{2}$ has an orthonormal basis given by the union of the sets

$$
\begin{aligned}
& \left\{e_{n}=\frac{\sqrt{3(n+1)}}{2 \sqrt{\left(1-(1 / 2)^{2 n+2}\right.}} z^{n}, n \geq 0\right\}, \\
& \left\{e_{-1}=\frac{\sqrt{3}}{\sqrt{8 \ln 2} z}\right\}, \text { and } \\
& \left\{e_{-n}=\frac{\sqrt{3(n-1)}}{2 \sqrt{\left(2^{2 n-2}-1\right)}} \frac{1}{z^{n}}, n \geq 2 .\right\} .
\end{aligned}
$$

We consider hyponormality of Toeplitz operators with a symbol of the form $f=$ $g_{1}+\overline{g_{2}}$, where $g_{1}$ and $g_{2}$ are bounded analytic functions on $C_{1 / 2}$. We begin by recalling some known properties of Toeplitz operators.

\section{Some BAsic PRoperties}

Lemma 2.1. Let $f$ and $g$ be bounded and measurable on $C_{1 / 2}$. The following properties hold:

a) $T_{f+g}=T_{f}+T_{g}$.

b) $T_{f}^{*}=T_{\bar{f}}$.

c) $T_{f} T_{g}=T_{f g}$ if $g$ is analytic on $C_{1 / 2}$ or $f$ is conjugate analytic.

d) $T_{\bar{f}} T_{f}-T_{f} T_{\bar{f}}=H_{\bar{f}}^{*} H_{\bar{f}}$ if $f$ is analytic.

The next proposition is easy to prove and its proof is omitted.

Proposition 2.2. Let $g_{1}$ and $g_{2}$ be polynomials. The following are equivalent:

a) $T_{g_{1}+\overline{g_{2}}}$ is hyponormal.

b) $T_{\overline{g_{2}}} T_{g_{2}}-T_{g_{2}} T_{\overline{g_{2}}} \leq T_{\overline{g_{1}}} T_{g_{1}}-T_{g_{1}} T_{\overline{g_{1}}}$.

c) $H_{\overline{g_{2}}}^{*} H_{\overline{g_{2}}} \leq H_{\overline{g_{1}}}^{*} H_{\overline{g_{1}}}$.

d) $H_{g_{2}}=K H_{g_{1}}$, where $K$ is an operator of norm less than one.

The following lemma provides computations that will be needed.

Lemma 2.3. The projection $P$ on $A_{1 / 2}^{2}$ satisfies the following relations:

1) $P\left(z^{m} \overline{z^{n}}\right)=\frac{(m-n+1)\left(1-(1 / 2)^{2 m+2}\right)}{(m+1)\left(1-(1 / 2)^{2 m-2 n+2}\right)} z^{m-n}$, if $m \geq n$.

2) $P\left(z^{m} \overline{z^{n}}\right)=\frac{(n-m-1)\left(1-(1 / 2)^{2 m+2)}\right)}{(m+1)\left(2^{2 n-2 m-2}-1\right)} \frac{1}{z^{n-m}}$, if $n \geq m+2$.

3) $P\left(z^{m} \overline{z^{m+1}}\right)=\frac{\left(1-(1 / 2)^{2 m+2}\right)}{2 \ln 2(m+1)} \frac{1}{z}$, if $n=m+1$.

4) $P\left(\frac{1}{z^{m}} \overline{z^{n}}\right)=\frac{\left.(m+n-1)\left(2^{2 m-2}-1\right)\right)}{\left(2^{2(m+n)-2}-1\right)(m-1)} \frac{1}{z^{m+n}}$, if $m \geq 2$. 
5) $P\left(\frac{1}{z} \overline{z^{n}}\right)=\frac{2 n \ln 2}{\left(2^{2 n}-1\right)} \frac{1}{z^{n+1}}$, if $n \geq 1$.

6) $P\left(\frac{1}{\overline{z^{m}}} z^{n}\right)=\frac{(m+n+1)\left(\left(1-(1 / 2)^{2 n+2}\right)\right.}{(n+1)\left(1-(1 / 2)^{2(m+n)+2}\right)} z^{m+n}$.

7) $P\left(\frac{1}{\overline{z^{m}} z^{n}}\right)=\frac{((m-n)+1)\left(2^{2 n-2}-1\right)}{(n-1)\left(1-(1 / 2)^{2(m-n)+2}\right)} z^{m-n}$, if $m \geq n, n \neq 1$.

8) $P\left(\frac{1}{\overline{z^{m} z}}\right)=\frac{2 m \ln 2}{\left(1-(1 / 2)^{2 m}\right)} z^{m-1}$, if $m \geq 1$.

9) $P\left(\frac{1}{\overline{z^{m}} z^{n}}\right)=\frac{(n-m-1)\left(2^{2 n-2}-1\right)}{(n-1)\left(2^{2(n-m)-2}-1\right)} \frac{1}{z^{n-m}}$, if $m \geq 1, n-m>1$.

10) $P\left(\frac{1}{\overline{z^{m}} z^{m+1}}\right)=\frac{\left(2^{2 m}-1\right)}{2 m \ln 2} \frac{1}{z}$, if $m \geq 1$.

\section{First MAIN RESUlt}

We begin with a matrix computation.

Lemma 3.1. Let $f=\sum_{1}^{\infty} a_{k} z^{k}$ be bounded on $C_{1 / 2}$. Then for $i, j \geq 1$ we have

$$
\begin{aligned}
\left\langle T_{\bar{f}} T_{f}-\right. & \left.T_{f} T_{\bar{f}}\left(e_{j}\right), e_{i}\right\rangle \\
= & \sum_{\substack{1 \leq k \\
1 \leq k+j-i}} \overline{a_{k+j-i}} a_{k} \frac{\sqrt{i+1} \sqrt{j+1}\left(1-(1 / 2)^{2(k+j)+2)}\right)}{\sqrt{1-(1 / 2)^{2 i+2}} \sqrt{1-(1 / 2)^{2 j+2}}(k+j+1)} \\
& -\sum_{\substack{1 \leq k \leq j \\
1 \leq k+i-j}} \overline{a_{k}} a_{k+i-j} \frac{(j-k+1) \sqrt{1-(1 / 2)^{2 i+2}} \sqrt{1-(1 / 2)^{2 j+2}}}{\left(1-(1 / 2)^{2(j-k)+2}\right) \sqrt{i+1} \sqrt{j+1}} \\
& -\overline{a_{j+1}} a_{i+1} \frac{\sqrt{\left(1-(1 / 2)^{2 i+2}\right.} \sqrt{\left(1-(1 / 2)^{2 j+2}\right.}}{2 \ln 2 \sqrt{i+1} \sqrt{j+1}} \\
& -\sum_{\substack{j+2 \leq k \\
1 \leq k+i-j}} \overline{a_{k}} a_{k+i-j} \frac{(k-i-1) \sqrt{\left(1-(1 / 2)^{2 i+2}\right.} \sqrt{\left(1-(1 / 2)^{2 j+2}\right.}}{\sqrt{i+1} \sqrt{j+1}}
\end{aligned} .
$$

Proof. We have

$$
\begin{aligned}
\left\langle T_{\bar{f}} T_{f}\left(e_{j}\right), e_{i}\right\rangle & =\sum_{k, l=1}^{\infty} \overline{a_{l}} a_{k} \frac{\sqrt{3(i+1)}}{2 \sqrt{\left(1-(1 / 2)^{2 i+2}\right.}} \frac{\sqrt{3(j+1)}}{2 \sqrt{\left(1-(1 / 2)^{2 j+2}\right.}}\left\langle z^{k+j}, z^{i+l}\right\rangle \\
& =\sum_{\substack{1 \leq k \\
1 \leq k+j-i}} \frac{\overline{a_{k+j-i}} a_{k}\left(1-(1 / 2)^{2(k+j)+2)}\right) \sqrt{(i+1)(j+1)}}{(k+j+1) \sqrt{\left(1-(1 / 2)^{2 i+2}\right)\left(1-(1 / 2)^{2 j+2}\right)}}
\end{aligned}
$$


Similarly, we get

$$
\begin{aligned}
\left\langle T_{f} T_{\bar{f}}\left(e_{j}\right), e_{i}\right\rangle= & \sum_{\substack{1 \leq k+i-j \\
1 \leq k \leq j}} \frac{\overline{a_{k}} a_{k+i-j}(j-k+1) \sqrt{1-(1 / 2)^{2 i+2}} \sqrt{1-(1 / 2)^{2 j+2}}}{\left(1-(1 / 2)^{2(j-k)+2} \sqrt{i+1} \sqrt{j+1}\right.} \\
& +\overline{a_{j+1}} a_{i+1} \frac{\sqrt{\left(1-(1 / 2)^{2 i+2}\right.}}{2 \ln 2 \sqrt{i+1}} \frac{\sqrt{\left(1-(1 / 2)^{2 j+2}\right.}}{\sqrt{j+1}} \\
& +\sum_{\substack{j+2 \leq k \\
1 \leq k+i-j}} \frac{\overline{a_{k}} a_{k+i-j}(k-j-1) \sqrt{1\left(-(1 / 2)^{2 i+2}\right)\left(1-(1 / 2)^{2 j+2}\right)}}{\sqrt{(i+1)(j+1)}} .
\end{aligned}
$$

Set $\beta_{i, j}=\left\langle T_{\bar{f}} T_{f}-T_{f} T_{\bar{f}}\left(e_{j}\right), e_{i}\right\rangle, i, j \geq 1$. By rewriting the expression for $\beta_{i, j}$ we obtain

$$
\begin{aligned}
\beta_{i+p, i}= & \sum_{\substack{1 \leq k \leq i \\
1 \leq k+p}} \overline{a_{k}} a_{k+p} \frac{\sqrt{i+1} \sqrt{i+p+1}\left(1-(1 / 2)^{2(k+p+i)+2)}\right)}{\sqrt{1-(1 / 2)^{2 i+2}} \sqrt{1-(1 / 2)^{2(i+p)+2}}(k+p+i+1)} \\
& -\sum_{\substack{1 \leq k \leq i \\
1 \leq k+p}} \overline{a_{k}} a_{k+p} \frac{(i-k+1) \sqrt{1-(1 / 2)^{2 i+2}} \sqrt{1-(1 / 2)^{2(i+p)+2}}}{\left(1-(1 / 2)^{2(i-k)+2}\right) \sqrt{i+1} \sqrt{i+p+1}} \\
& +\overline{a_{i+1}} a_{i+p+1} \frac{\sqrt{i+1} \sqrt{i+p+1}\left(1-(1 / 2)^{2(2 i+1+p)+2)}\right)}{\sqrt{1-(1 / 2)^{2 i+2}} \sqrt{1-(1 / 2)^{2(i+p)+2}}(2(i+1)+p)} \\
& -\overline{a_{i+1}} a_{i+p+1} \frac{\sqrt{\left(1-(1 / 2)^{2 i+2}\right.} \sqrt{\left(1-(1 / 2)^{2(i+p)+2}\right.}}{2 \ln 2 \sqrt{i+1} \sqrt{i+p+1}} \\
& +\sum_{i+2 \leq k} \overline{a_{k}} a_{k+p} \frac{\sqrt{i+1} \sqrt{i+p+1}\left(1-(1 / 2)^{2(k+p+i)+2)}\right)}{\sqrt{1-(1 / 2)^{2 i+2}} \sqrt{1-(1 / 2)^{2(i+p)+2}}(k+p+i+1)} \\
& -\sum_{i+2 \leq k} \overline{a_{k}} a_{k+p} \frac{(k-i-1) \sqrt{\left(1-(1 / 2)^{2 i+2}\right.} \sqrt{\left(1-(1 / 2)^{2(i+p)+2}\right.}}{\sqrt{i+1} \sqrt{i+p+1}} \\
= & \sum_{1 \leq k \leq i} \overline{a_{k}} a_{k+p} Q_{i, k, p}+\overline{a_{i+1}} a_{i+p+1} R_{i, p}+\sum_{i+2 \leq k} \overline{a_{k}} a_{k+p} S_{i, k, p} .
\end{aligned}
$$

Lemma 3.2. We have $\lim _{i \rightarrow \infty} i^{2} \beta_{i+p, i}=\gamma_{i+p, i}$, where $\left(\gamma_{i, j}\right)$ is the matrix of the Hardy space Topelitz operator $T_{\left|f^{\prime}\right|^{2}}$.

Proof. An elementary computation shows that $\lim _{i \rightarrow \infty} i^{2} Q_{i, k, p}=k(k+p)$. Set $h_{i}(k)=i^{2} \chi_{\{1, \ldots, i\}}(k) \overline{a_{k}} a_{k+p} Q_{i, k, p}$. The first sum in the above expression of $\beta_{i+p, i}$ can be written as $\int h_{i}(k) d \mu(k)$, where $d \mu$ is the counting measure. It is easy to see that for $i$ sufficiently large, $\left|h_{i}(k)\right| \leq 2\left|a_{k} a_{k+p}\right| \leq k^{2}\left|a_{k}\right|^{2}+(k+p)^{2}\left|a_{k+p}\right|^{2}=$ $M(k)$. Since $f^{\prime} \in H^{2}$, the function $M(k)$ is integrable with respect to the counting measure. 
By the dominated convergence theorem we obtain:

$$
\lim _{i \rightarrow \infty} i^{2} \sum_{\substack{1 \leq k \leq i \\ 1 \leq k+p}} \overline{a_{k}} a_{k+p} Q_{i, k, p}=\sum k(k+p) \overline{a_{k}} a_{k+p} .
$$

Also, for $i$ large, there exists a constant $C$ such that

$$
\left|i^{2} \overline{a_{i+1}} a_{i+p+1} R_{i, p}\right| \leq C\left((i+1)^{2}\left|a_{i+1}\right|^{2}+(i+p+1)^{2}\left|a_{i+p+1}\right|^{2}\right) .
$$

Thus $\lim _{i \rightarrow \infty} i^{2} \overline{a_{i+1}} a_{i+p+1} R_{i, p}=0$. Finally, it is not difficult to see that $i^{2}\left|S_{i, k, p}\right| \leq k(k+p)$. Using the dominated convergence theorem we obtain

$$
\lim _{i \rightarrow \infty} i^{2} \sum_{i+2 \leq k} \overline{a_{k}} a_{k+p} S_{i, k, p}=0 .
$$

We deduce that $\lim _{i \rightarrow \infty} i^{2} \beta_{i+p, i}=\sum k(k+p) \overline{a_{k}} a_{k+p}$ and recognize this last limit as being equal to $\gamma_{i+p, i}$, where $\left(\gamma_{i, j}\right)$ is the matrix of the Hardy space Toeplitz operator $T_{\left|f^{\prime}\right|^{2}}$.

We are led to the following necessary condition for hyponormality.

Theorem 3.3. Let $f=\sum_{1}^{\infty} a_{k} z^{k}$ and $g=\sum_{1}^{\infty} b_{k} z^{k}$ be bounded on $C_{1 / 2}$. Assume that $f^{\prime} \in H^{2}$. If $T_{f+\bar{g}}$ is hyponormal then $g^{\prime} \in H^{2}$ and $\left|g^{\prime}\right| \leq\left|f^{\prime}\right|$ a.e. on the unit circle.

Proof. If $\left(\theta_{i, j}\right)$ denotes the matrix of $T_{\bar{f}} T_{f}-T_{f} T_{\bar{f}}-T_{\bar{g}} T_{g}-T_{g} T_{\bar{g}}$ and $\left(\sigma_{i, j}\right)$ denotes the matrix of $T_{\bar{g}} T_{g}-T_{g} T_{\bar{g}}$, then the inequality $\sigma_{i, i} \leq \beta_{i, i}$ leads to

$$
\begin{aligned}
\sum_{1 \leq k \leq i}\left|b_{k}\right|^{2} Q_{i, k, 0}+\left|b_{i+1}\right|^{2} & R_{i, 0}+\sum_{i+2 \leq k}\left|b_{k}\right|^{2} S_{i, k, 0} \\
\leq & \sum_{1 \leq k \leq i}\left|a_{k}\right|^{2} Q_{i, k, 0}+\left|a_{i+1}\right|^{2} R_{i, 0}+\sum_{i+2 \leq k}\left|a_{k}\right|^{2} S_{i, k, 0} .
\end{aligned}
$$

We deduce that $\sum_{1 \leq k \leq i} i^{2}\left|b_{k}\right|^{2} Q_{i, k, 0} \leq i^{2} \beta_{i, i}$. Since $\lim _{i \rightarrow \infty} i^{2} Q_{i, k, 0}=k^{2}$, writing the left hand side of this last inequality as an integral with respect to the counting measure and using Fatou's lemma we get $\sum k^{2}\left|b_{k}\right|^{2} \leq \sum k^{2}\left|a_{k}\right|$ and $g^{\prime} \in H^{2}$. From the previous lemma, $\lim _{i \rightarrow \infty} i^{2} \theta_{i+p, i}=\lambda_{i+p, i}$, where $\left(\lambda_{i, j}\right)$ denotes the matrix of

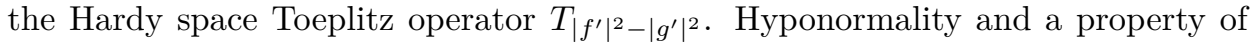
Toeplitz matrices [6] lead to $\left|g^{\prime}\right| \leq\left|f^{\prime}\right|$ a.e. on the unit circle.

Corollary 3.4. Let $f=\sum_{1}^{\infty} a_{k} z^{k}$ and $g=\sum_{1}^{\infty} b_{k} z^{k}$ be analytic and univalent in an open set containing $C_{1 / 2}$. Then $T_{f+\bar{g}}$ is normal if and only if $g=c f$, where $c$ is a constant with $|c|=1$.

Proof. Only the necessary condition needs to be shown. Normality implies that $\left|g^{\prime}\right|=\left|f^{\prime}\right|$ on the unit circle. Thus $f^{\prime}$ and $g^{\prime}$ have the same finite number of zeros (if any) with the same multiplicity. We thus have $\frac{\left|f^{\prime}\right|}{\left|g^{\prime}\right|}=\frac{\left|g^{\prime}\right|}{\left|f^{\prime}\right|}=1$ on the unit circle. By the maximum principle, $g^{\prime}=c f^{\prime}$ with $|c|=1$. We get $g=c f$. 
Lemma 3.5. Let $f=\sum_{1}^{\infty} a_{k} z^{k}$ be bounded on $C_{1 / 2}$. Then for $i \geq 3, j \geq 3$ we have

$$
\begin{aligned}
\left\langle T_{\bar{f}} T_{f}-\right. & \left.T_{f} T_{\bar{f}}\left(e_{-j}\right), e_{-i}\right\rangle \\
= & \sum_{\substack{1 \leq k<j-1 \\
1 \leq k+i-j}} \overline{a_{k+i-j}} a_{k} \frac{\sqrt{(i-1)}}{\sqrt{\left(2^{2 i-2}-1\right)}} \frac{\sqrt{(j-1)}}{\sqrt{\left(2^{2 j-2}-1\right)}} \frac{\left(2^{2(j-k)-2}-1\right)}{(j-k-1)} \\
& +2 \ln 2 \overline{a_{i-1}} a_{j-1} \frac{\sqrt{i-1}}{\sqrt{2^{2 i-2}-1}} \frac{\sqrt{j-1}}{\sqrt{2^{2 j-2}-1}} \\
& +\sum_{j \leq k} \overline{a_{k+i-j}} a_{k} \frac{\sqrt{(i-1)}}{\sqrt{\left(2^{2 i-2}-1\right)}} \frac{\sqrt{(j-1)}}{\sqrt{\left(2^{2 j-2}-1\right)}} \frac{\left(1-(1 / 2)^{2(k-j)+2}\right)}{k-j+1} \\
& -\sum_{\substack{1 \leq k \\
1 \leq k+j-i}} \overline{a_{k}} a_{k+j-i} \frac{(k+j-1) \sqrt{\left(2^{2 i-2}-1\right)} \sqrt{2^{2 j-2}-1}}{\left(2^{2(j+k)-2}-1\right) \sqrt{i-1}} \sqrt{j-1}
\end{aligned}
$$

Proof. We have

$$
\begin{aligned}
\left\langle T_{\bar{f}} T_{f}\left(e_{-j}\right), e_{-i}\right\rangle= & \sum_{\substack{1 \leq k<j-1 \\
1 \leq k+i-j}} \overline{a_{k+i-j}} a_{k} \frac{\sqrt{i-1}}{\sqrt{2^{2 i-2}-1}} \frac{\sqrt{j-1}}{\sqrt{2^{2 j-2}-1}} \frac{\left(2^{2(j-k)-2}-1\right)}{(j-k-1)} \\
& +2 \ln 2 \overline{a_{i-1}} a_{j-1} \frac{\sqrt{i-1}}{\sqrt{2^{2 i-2}-1}} \frac{\sqrt{j-1}}{\sqrt{2^{2 j-2}-1}} \\
& +\sum_{j \leq k} \overline{a_{k+i-j}} a_{k} \frac{\sqrt{(i-1)}}{\sqrt{\left(2^{2 i-2}-1\right)}} \frac{\sqrt{(j-1)}}{\sqrt{\left(2^{2 j-2}-1\right)}} \frac{\left(1-(1 / 2)^{2(k-j)+2}\right)}{k-j+1} .
\end{aligned}
$$

Similarly,

$$
\begin{aligned}
\left\langle T_{f} T_{\bar{f}}\left(e_{-j}\right), e_{-i}\right\rangle & =\sum_{k, l=1}^{\infty} \overline{a_{k}} a_{l} \frac{\sqrt{3(i-1)}}{2 \sqrt{\left(2^{2 i-2}-1\right)}} \frac{\sqrt{3(j-1)}}{2 \sqrt{\left(2^{2 j-2}-1\right)}}\left\langle P\left(\overline{z^{k}} \frac{1}{z^{j}}\right), P\left(\overline{z^{l}} \frac{1}{z^{i}}\right)\right\rangle \\
& =\sum_{\substack{1 \leq k \\
1 \leq k+j-i}} \overline{a_{k}} a_{k+j-i} \frac{(k+j-1) \sqrt{2^{2 i-2}-1} \sqrt{2^{2 j-2}-1}}{\left(2^{2(j+k)-2}-1\right) \sqrt{i-1} \sqrt{j-1}} .
\end{aligned}
$$

Let $\beta_{-i,-j}=\left\langle\left(T_{\bar{f}} T_{f}-T_{f} T_{\bar{f}}\right)\left(e_{-j}\right), e_{-i}\right\rangle$ and denote by $\left(\zeta_{i, j}\right)$ the matrix of the Toeplitz operator $T_{\left|f_{1 / 2}^{\prime}\right|^{2}}$ on the Hardy space of the unit disk, where $f_{1 / 2}(z)=$ $\sum \overline{a_{k}} \frac{z^{k}}{2^{k}}$.

We can show the following lemma. 
Lemma 3.6. We have $\lim _{i \rightarrow \infty} i^{2} \beta_{-i-p,-i}=\zeta_{i+p, i}$.

Proof.

$$
\begin{aligned}
& \beta_{-i-p,-i} \\
& =\sum_{\substack{1 \leq k<i-1 \\
1 \leq k+p}} \overline{a_{k+p}} a_{k} \frac{\sqrt{(i-1)}}{\sqrt{\left(2^{2 i-2}-1\right)}} \frac{\sqrt{(i+p-1)}}{\sqrt{\left(2^{2(i+p)-2}-1\right)}} \frac{\left(2^{2(i-k)-2}-1\right)}{(i-k-1)} \\
& +2 \ln 2 \overline{a_{i+p-1}} a_{i-1} \frac{\sqrt{i+p-1}}{\sqrt{2^{2(i+p)-2}-1}} \frac{\sqrt{i-1}}{\sqrt{2^{2 i-2}-1}} \\
& +\sum_{i \leq k} \overline{a_{k+p}} a_{k} \frac{\sqrt{i-1}}{\sqrt{\left(2^{2 i-2}-1\right)}} \frac{\sqrt{i+p-1}}{\sqrt{\left(2^{2(i+p)-2}-1\right)}} \frac{\left(1-(1 / 2)^{2(k-i)+2}\right)}{k-i+1} \\
& -\sum_{\substack{1 \leq k \\
1 \leq k+p}} \overline{a_{k+p}} a_{k} \frac{(k+p+i-1) \sqrt{\left(2^{2 i-2}-1\right)} \sqrt{2^{2(i+p)-2}-1}}{\left(2^{2(i+k+p)-2}-1\right) \sqrt{i-1} \sqrt{i+p-1}} \\
& =\sum_{\substack{1 \leq k<i-1 \\
1 \leq k+p}} \frac{\overline{a_{k+p}} a_{k}(i-1)(i+p-1)\left(2^{2(i-k)-2}-1\right)\left(2^{2(i+k+p)-2}-1\right)}{\sqrt{\left(2^{2 i-2}-1\right)\left(2^{2(i+p)-2}-1\right)} \sqrt{(i-1)(i+p-1)}(i-k-1)\left(2^{2(i+k+p)-2}-1\right)} \\
& -\sum_{\substack{1 \leq k<i-1 \\
1 \leq k+p}} \frac{\overline{a_{k+p}} a_{k}(k+p+i-1)(i-k-1)\left(2^{2 i-2}-1\right)\left(2^{2(i+p)-2}-1\right)}{\sqrt{\left(2^{2 i-2}-1\right)\left(2^{2(i+p)-2}-1\right)} \sqrt{(i-1)(i+p-1)}(i-k-1)\left(2^{2(i+k+p)-2}-1\right)} \\
& +\overline{a_{i+p-1}} a_{i-1}\left(2 \ln 2 \frac{\sqrt{i-1}}{\sqrt{2^{2 i-2}-1}} \frac{\sqrt{i+p-1}}{\sqrt{2^{2(i+p)-2}-1}}\right. \\
& \left.-\frac{(2 i-2+p) \sqrt{\left(2^{2 i-2}-1\right)} \sqrt{2^{2(i+p)-2}-1}}{\left(2^{2(2 i-1+p)-2}-1\right) \sqrt{i-1} \sqrt{i+p-1}}\right) \\
& +\sum_{i \leq k} \overline{a_{k+p}} a_{k}\left(\frac{\sqrt{i-1}}{\sqrt{\left(2^{2 i-2}-1\right)}} \frac{\sqrt{i+p-1}}{\sqrt{\left(2^{2(i+p)-2}-1\right)}} \frac{\left(1-(1 / 2)^{2(k-i)+2}\right)}{k-i+1}\right) \\
& -\sum_{i \leq k} \overline{a_{k+p}} a_{k}\left(\frac{(k+p+i-1) \sqrt{\left(2^{2 i-2}-1\right)} \sqrt{2^{2(i+p)-2}-1}}{\left(2^{2(i+k+p)-2}-1\right) \sqrt{i-1} \sqrt{i+p-1}}\right) \\
& =\sum_{\substack{1 \leq k<i-1 \\
1 \leq k+p}} \overline{a_{k+p}} a_{k} Q_{i, p, k}^{\prime}+\overline{a_{i+p-1}} a_{i-1} R_{i, p}^{\prime}+\sum_{i \leq k} \overline{a_{k+p}} a_{k} S_{i, k, p}^{\prime} .
\end{aligned}
$$

A computation shows that $\lim _{i \rightarrow \infty} i^{2} Q_{i, p, k}^{\prime}=\frac{1}{2^{2 k+p}}$. As in the proof of the previous theorem we can show that

$$
\lim _{i \rightarrow \infty} i^{2} \sum_{\substack{1 \leq k<i-1 \\ 1 \leq k+p}} \overline{a_{k+p}} a_{k} Q_{i, p, k}^{\prime}=\sum_{\substack{1 \leq k \\ 1 \leq k+p}} k(k+p) \frac{a_{k}}{2^{k}} \frac{\overline{a_{k+p}}}{2^{k+p}} .
$$

We see that this last limit is equal to $\zeta_{i, i+p}$. We also show that

$$
\lim _{i \rightarrow \infty} i^{2} \overline{a_{i+p-1}} a_{i-1} R_{i, p}^{\prime}=0
$$


and

$$
\lim _{i \rightarrow \infty} i^{2} \sum_{i \leq k} \overline{a_{k+p}} a_{k} S_{i, k, p}^{\prime}=0
$$

We deduce that

$$
\lim _{i \rightarrow \infty} i^{2} \beta_{-i-p,-i}=\zeta_{i+p, i}
$$

If $f=\sum_{1}^{\infty} a_{k} z^{k}$ is bounded analytic on $C_{1 / 2}$, then clearly $\sum \frac{k^{2}}{2^{2 k}}\left|a_{k}\right|^{2}<\infty$. We can also see that $\left|g_{1 / 2}^{\prime}\right| \leq\left|f_{1 / 2}^{\prime}\right|$ a.e. on the unit circle is equivalent to $\left|g^{\prime}\right| \leq\left|f^{\prime}\right|$ a.e. on $\{z:|z|=1 / 2\}$.

Theorem 3.7. Let $f=\sum_{1}^{\infty} a_{k} z^{k}$ and $g=\sum_{1}^{\infty} b_{k} z^{k}$ be bounded on $C_{1 / 2}$. If $T_{f+\bar{g}}$ is hyponormal then $\left|g^{\prime}\right| \leq\left|f^{\prime}\right|$ a.e. on $\{z:|z|=1 / 2\}$.

The proof is similar to the proof of the previous theorem and is omitted. Combining the previous two theorems we get our first main result.

Theorem 3.8. Let $f=\sum_{1}^{\infty} a_{k} z^{k}$ and $g=\sum_{1}^{\infty} b_{k} z^{k}$ be bounded on $C_{1 / 2}$ and assume that $f^{\prime} \in H^{2}$. If $T_{f+\bar{g}}$ is hyponormal then $g^{\prime} \in H^{2}$ and $\left|g^{\prime}\right| \leq\left|f^{\prime}\right|$ a.e. on $\{z:|z|=1\} \cup\{z:|z|=1 / 2\}$.

\section{SECOND MAIn RESUlt}

We now put $f=\sum_{1}^{\infty} a_{k} \frac{1}{z^{k}}$ and $g=\sum_{1}^{\infty} b_{k} \frac{1}{z^{k}}$ and assume that $f$ and $g$ are bounded on $C_{1 / 2}$. We need the following computation.

Lemma 4.1. For $i \geq 1, j \geq 1$ we have

$$
\begin{aligned}
\left\langle T_{\bar{f}} T_{f}\right. & \left.-T_{f} T_{\bar{f}}\left(e_{j}\right), e_{i}\right\rangle \\
= & \sum_{1 \leq k, k+i-j} \overline{a_{k+i-j}} a_{k} \frac{\sqrt{(i+1)} \sqrt{(j+1)}\left(1-(1 / 2)^{2(j-k)+2}\right)}{\sqrt{\left(1-(1 / 2)^{2 i+2}\right.} \sqrt{\left(1-(1 / 2)^{2 j+2}\right.}(j-k+1)} \\
& -\sum_{1 \leq k, k+j-i} \overline{a_{k}} a_{k+j-i} \frac{\sqrt{\left(1-(1 / 2)^{2 i+2}\right)} \sqrt{\left(1-(1 / 2)^{2 j+2}\right.}(j+k+1)}{\sqrt{i+1} \sqrt{j+1}\left(1-(1 / 2)^{2(j+k)+2}\right)} .
\end{aligned}
$$

Proof. We have

$$
\begin{aligned}
\left\langle T_{\bar{f}} T_{f}\left(e_{j}\right), e_{i}\right\rangle & =\sum_{k, l=1}^{\infty} \overline{a_{l}} a_{k} \frac{\sqrt{3(i+1)}}{2 \sqrt{\left(1-(1 / 2)^{2 i+2}\right.}} \frac{\sqrt{3(j+1)}}{2 \sqrt{\left(1-(1 / 2)^{2 j+2}\right.}}\left\langle z^{j-k}, z^{i-l}\right\rangle \\
& =\sum_{1 \leq k, k+i-j}^{\infty} \overline{a_{k+i-j}} a_{k} \frac{\sqrt{(i+1)}}{\sqrt{\left(1-(1 / 2)^{2 i+2}\right.}} \frac{\sqrt{(j+1)}}{\sqrt{\left(1-(1 / 2)^{2 j+2}\right.}} \frac{\left(1-(1 / 2)^{2(j-k)+2}\right.}{j-k+1}
\end{aligned}
$$


and

$$
\begin{aligned}
\left\langle T_{f} T_{\bar{f}}\left(e_{j}\right), e_{i}\right\rangle= & \sum_{k, l=1}^{\infty} \overline{a_{k}} a_{l} \frac{\sqrt{3(i+1)}}{2 \sqrt{\left(1-(1 / 2)^{2 i+2}\right.}} \frac{\sqrt{3(j+1)}}{2 \sqrt{\left(1-(1 / 2)^{2 j+2}\right.}} \\
& \times\left\langle P\left(\frac{1}{\overline{z^{k}}} z^{j}\right), P\left(\frac{1}{\overline{z^{l}}} z^{i}\right)\right\rangle \\
= & \sum_{1 \leq k, k+j-i}^{\infty} \overline{a_{k}} a_{k+j-i} \frac{\sqrt{\left(1-(1 / 2)^{2 i+2}\right)} \sqrt{\left(1-(1 / 2)^{2 j+2}\right.}(j+k+1)}{\sqrt{i+1} \sqrt{j+1}\left(1-(1 / 2)^{2(j+k)+2}\right)} .
\end{aligned}
$$

We get, using the same notations as before,

$$
\begin{aligned}
\beta_{i+p, i}= & \sum_{\substack{1 \leq k-p \\
1 \leq k}} \overline{a_{k}} a_{k-p} \frac{\sqrt{(i+1)} \sqrt{(i+p+1)}\left(1-(1 / 2)^{2(i-k+p)+2}\right)}{\sqrt{\left(1-(1 / 2)^{2 i+2}\right.} \sqrt{\left(1-(1 / 2)^{2(i+p)+2}\right.} i-k+p+1} \\
& -\sum_{\substack{1 \leq k \\
1 \leq k-p}} \overline{a_{k}} a_{k-p} \frac{\sqrt{\left(1-(1 / 2)^{2 i+2}\right)} \sqrt{\left(1-(1 / 2)^{2(i+p)+2}\right.}(i+k+1)}{\sqrt{i+1} \sqrt{i+p+1}\left(1-(1 / 2)^{2(i+k)+2}\right)} \\
= & \sum_{\substack{1 \leq k \\
1 \leq k-p}} \overline{a_{k}} a_{k-p} U_{i, k, p} .
\end{aligned}
$$

A computation shows that

$$
\lim _{i \rightarrow \infty} i^{2} \beta_{i+p, i}=\sum_{1 \leq k, k-p} \overline{a_{k}} a_{k-p} k(k-p) .
$$

We recognize the general element $\xi_{m+p, m}$ of the matrix of the Toeplitz operator $T_{\left|\tilde{f}^{\prime}\right|}$ on the Hardy space of the unit disk with $\widetilde{f}$ defined by $\widetilde{f(z)}=\sum_{1}^{\infty} \overline{a_{k}} z^{k}$. Obviously the condition $\left|\widetilde{g}^{\prime}\left(e^{i \theta}\right)\right| \leq\left|\tilde{f}^{\prime}\left(e^{i \theta}\right)\right|$ a.e. on the unit circle is the same as $\left|g^{\prime}\right| \leq\left|f^{\prime}\right|$ a.e. on the unit circle. The condition $\tilde{f}^{\prime} \in H^{2}$ is equivalent to $\sum k^{2}\left|a_{k}\right|^{2}<\infty$ and this is satisfied if $f=\sum_{1}^{\infty} a_{k} \frac{1}{z^{k}}$ is bounded on $C_{1 / 2}$. Using similar methods we obtain the following theorem.

Theorem 4.2. Let $f=\sum_{1}^{\infty} a_{k} \frac{1}{z^{k}}$ and $g=\sum_{1}^{\infty} b_{k} \frac{1}{z^{k}}$ be analytic and bounded on $C_{1 / 2}$. If $T_{f+\bar{g}}$ is hyponormal then $\left|g^{\prime}\right| \leq\left|f^{\prime}\right|$ a.e. on the unit circle.

If we set $f_{2}(z)=\sum 2^{k} a_{k} z^{k}$, then $f_{2}^{\prime} \in H^{2}$ is equivalent to $\sum k^{2} 2^{2 k}\left|a_{k}\right|^{2}<\infty$. In this case, $\left|g_{2}^{\prime}\right| \leq\left|f_{2}^{\prime}\right|$ a.e. on the unit circle is equivalent to $\left|g^{\prime}\right| \leq\left|f^{\prime}\right|$ a.e. on $\{z:|z|=1 / 2\}$. Let $\left(\rho_{i, j}\right)$ denote the matrix of the Hardy space Toeplitz operator $T_{\left|f_{2}^{\prime}\right|^{2}}$. Using the same notations we can show the following lemma, the proof of which is omitted.

Lemma 4.3. $\lim _{i \rightarrow \infty} i^{2} \beta_{-i-p,-i}=\rho_{i+p, i}$.

We obtain our second main result. 
Theorem 4.4. Let $f=\sum_{1}^{\infty} a_{k} \frac{1}{z^{k}}$ and $g=\sum_{1}^{\infty} b_{k} \frac{1}{z^{k}}$ be bounded on $C_{1 / 2}$, with $\sum k^{2} 2^{2 k}\left|a_{k}\right|^{2}<\infty$. If $T_{f+\bar{g}}$ is hyponormal then $\sum k^{2} 2^{2 k}\left|b_{k}\right|^{2}<\infty$ and $\left|g^{\prime}\right| \leq\left|f^{\prime}\right|$ a.e. on $\{z:|z|=1\} \cup\{z:|z|=1 / 2\}$.

An application of the maximum modulus principle allows us to describe the normality of $T_{f+\bar{g}}$ under the condition of univalence.

Corollary 4.5. Let $f=\sum_{1}^{\infty} a_{k} \frac{1}{z^{k}}$ and $g=\sum_{1}^{\infty} b_{k} \frac{1}{z^{k}}$ be analytic and univalent in an open set containing $C_{1 / 2}$. Then $T_{f+\bar{g}}$ is normal if and only if $g=c f$, where $c$ is a constant with $|c|=1$.

We list two more results which are shown using methods similar to the ones used for the previous theorems.

Theorem 4.6. Let $f=\sum_{1}^{\infty} a_{k} z^{k}$ and $g=\sum_{1}^{\infty} b_{k} \frac{1}{z^{k}}$ be bounded on $C_{1 / 2}$. Assume that $\sum k^{2}\left|a_{k}\right|^{2}<\infty$. If $T_{f+\bar{g}}$ is hyponormal then $\sum k^{2}\left|b_{k}\right|^{2}<\infty$ and $\left|g^{\prime}\left(e^{i \theta}\right)\right| \leq$ $\left|f^{\prime}\left(e^{i \theta}\right)\right|$ a.e. on the unit circle.

Corollary 4.7. Let $f=\sum_{1}^{\infty} a_{k} z^{k}$ and $g=\sum_{1}^{\infty} b_{k} \frac{1}{z^{k}}$ be bounded on $C_{1 / 2}$. Assume that $f$ and $\widetilde{g}$ are univalent in an open set containig $C_{1 / 2}$. Then $T_{f+\bar{g}}$ is normal if and only if $\widetilde{g}=c f$ for some constant $c$ with $|c|=1$.

Theorem 4.8. Let $f=\sum_{1}^{\infty} a_{k} z^{k}$ and $g=\sum_{1}^{\infty} b_{k} \frac{1}{z^{k}}$ be bounded on $C_{1 / 2}$. If $T_{f+\bar{g}}$ is hyponormal then $\sum k^{2} 2^{2 k}\left|b_{k}\right|^{2}<\infty$ and $\left|g^{\prime}\left(\frac{1}{2} e^{i \theta}\right)\right| \leq\left|f^{\prime}\left(\frac{1}{2} e^{i \theta}\right)\right|$ for almost all $\theta$.

Corollary 4.9. Let $f=\sum_{1}^{\infty} a_{k} z^{k}$ and $g=\sum_{1}^{\infty} b_{k} \frac{1}{z^{k}}$ be bounded on $C_{1 / 2}$ and assume that $T_{f+\bar{g}}$ is hyponormal. The following holds:

i) $\sum k^{2} 2^{2 k}\left|b_{k}\right|^{2}<\infty$ and $\left|g^{\prime}\left(\frac{1}{2} e^{i \theta}\right)\right| \leq\left|f^{\prime}\left(\frac{1}{2} e^{i \theta}\right)\right|$ for almost all $\theta$.

ii) If $f^{\prime} \in H^{2}$ then $\left|g^{\prime}\left(e^{i \theta}\right)\right| \leq\left|f^{\prime}\left(e^{i \theta}\right)\right|$ a.e. on the unit circle.

\section{REFERENCES}

[1] P. Ahern and Z. Čučković, A mean value inequality with applications to Bergman space operators, Pacific J. Math. 173 (1996), no. 2, 295-305. MR 1394391.

[2] S. Axler, Bergman spaces and their operators, in Surveys of some recent results in operator theory, Vol. I, 1-50, Pitman Res. Notes Math. Ser., 171, Longman Sci. Tech., Harlow, 1988. MR 0958569

[3] C. C. Cowen, Hyponormal and subnormal Toeplitz operators, in Surveys of some recent results in operator theory, Vol. I, 155-167, Pitman Res. Notes Math. Ser., 171, Longman Sci. Tech., Harlow, 1988. MR 0958573 .

[4] C. C. Cowen, Hyponormality of Toeplitz operators, Proc. Amer. Math. Soc. 103 (1988), no. 3, 809-812. MR 0947663

[5] Z. Čučković and R. E. Curto, A new necessary condition for the hyponormality of Toeplitz operators on the Bergman space, J. Operator Theory 79 (2018), no. 2, 287-300. MR 3803558

[6] U. Grenander and G. Szegö, Toeplitz forms and their applications, California Monographs in Mathematical Sciences, University of California Press, Berkeley, 1958. MR 0094840

[7] I. S. Hwang, Hyponormality of Toeplitz operators on the Bergman space, J. Korean Math. Soc. 45 (2008), no. 4, 1027-1041. MR 2422725 
[8] H. Sadraoui, Hyponormality of Toeplitz operators and composition operators, Thesis (Ph.D.)Purdue University, 1992. MR 2687747

[9] H. Sadraoui and M. Guediri, Hyponormal Toeplitz operators on the Bergman space, Oper. Matrices 11 (2017), no. 3, 669-677. MR 3655674.

\author{
Houcine Sadraoui ${ }^{凶}$ \\ Mathematics Department, King Saud University, Riyadh, Saudi Arabia \\ sadrawi@ksu.edu.sa \\ Mohammed Guediri \\ Mathematics Department, King Saud University, Riyadh, Saudi Arabia \\ mguediri@ksu.edu.sa
}

Received: March 13, 2019

Accepted: August 26, 2019 